\title{
MEASUREMENTS OF THE SURFACE BRIGHTNESS OF THE EARTHSHINE WITH APPLICATIONS TO CALIBRATE LUNAR FLASHES
}

\author{
Pilar Montañés-Rodríguez and Enric Pallé \\ Instituto de Astrofísica de Canarias, C/ Vía Láctea s/n, E-38200 La Laguna, Tenerife, Spain; pmr@iac.es \\ AND \\ P. R. Goode \\ Big Bear Solar Observatory, New Jersey Institute of Technology, Newark, NJ 07102, USA \\ Received 2007 January 15; accepted 2007 May 9
}

\begin{abstract}
We have used the large database of photometric observations of the bright and dark portions of the face of the Moon from the Earthshine Project at Big Bear Solar Observatory to determine the surface brightness of the earthshine and its variations. Our purpose is to make these observations appropriate for the calibration of lunar flashes according to their magnitude. We have evaluated the daily, seasonal, and annual changes in magnitude for our entire data set and have also calibrated the surface brightness of the entire lunar geography for several lunar phases by means of the observation of lunar eclipses. We find variations between +12 and $+17 m_{V} \operatorname{arcsec}^{-2}$ with hourly changes upward of the order $0.25 m_{V} \operatorname{arcsec}^{-2}$, which are uniquely due to the terrestrial meteorology. This rapid change in the terrestrial flux reaching the Moon is usually neglected when calibrating the magnitude of lunar impact events. We justify this using earthshine observations to determine the brightness for the day, time, and selenographic location of a given event in order to improve the accuracy of its brightness calibration up to 0.25 mag.
\end{abstract}

Key words: atmospheric effects — Earth — Moon — techniques: photometric

\section{INTRODUCTION}

The brightness of the earthshine, or ashen light, reflected back to Earth from the dark portion of the face of the Moon, is strongly dependent on the amount of solar flux reflected by the Earth in the direction of the Moon (terrestrial flux; $F_{T}$ ). Once $F_{T}$ reaches the lunar surface, its retroflection will depend on the Moon's reflectivity, which strongly changes from lunar maria, whose main component is basalt having a relatively low albedo, to the lunar highlands, formed mainly by anorthosites of higher albedo (Zou et al. 2004). As the Moon waxes, it sees less and less of the sunlit face of the Earth, and thus has less and less $F_{T}$ falling on it, which introduces a strong modulation of the earthshine with lunar phase. Changes in orbital parameters, such as the Earth-Moon distance, will also have an impact on the earthshine brightness. Furthermore, $F_{T}$ is also affected by the cloudiness of the earthshinecontributing area of Earth. From one day to the next, the global cloud patterns on Earth change and so does the Earth's albedo, especially with the seasonal changes in snow/ice cover and orbital tilt of the Earth. Ultimately, $F_{T}$ will also change appreciably as the Earth rotates and different land, sea, and cloud patterns are visible from the Moon. The largest changes from night to night are due to the changing scattering angle of the earthshine.

The flux $F_{T}$ that reaches the Moon is sometimes considered to be constant. However, it may affect the study of lunar flashes when their brightness is determined by comparing the magnitude of the lunar region where a flash takes place to a reference star, especially if the observations of the flash and the star are not simultaneous.

Lunar flashes have several causes. The ones of major scientific interest are caused by impacts, a fundamental process of planetary evolution. The Moon presents an ideal record of the geological evolution of the Earth because its early surface is well preserved. The study of lunar impacts provides a good measure of their rate over terrestrial geological time, as well as of the rate, size, and velocity of present and future impacts on the Earth and the Moon. Understanding lunar impacts involves laboratory hypervelocity impact experiments and theoretical modeling. Models can be compared with field studies of terrestrial impact craters. However, the observation of an impact itself provides a direct measure of the released kinetic energy, by means of accurately quantifying the luminosity flux of the event, and if possible its light curve, as received on Earth (Bellot Rubio et al. 2000).

From the Earth, the observations of lunar impacts are typically carried out using a small telescope, with a modestly wide field optical system and a sensitive CCD (with very small pixel size and quick read-out time), or preferably a high-quality, low-level video camera (Ortiz et al. 2006). Ideally, one event should be simultaneously detected by more than one telescope to minimize instrumental noise. The brightness calibration of a lunar flash usually requires comparison with a standard star in the field of view of the dark of the Moon. To reduce the effect of the varying local atmosphere (clouds thickness) and small-scale changes in the terrestrial albedo, the calibration star should ideally be observed simultaneously with the impact. Reported lunar impact flashes seem to have a peak magnitude ranging between 5 (Ortiz et al. 2002 ) and $9.2^{1}$ visual magnitudes, with the latter limit depending on the instrumentation.

Lunar impacts are being measured not only from the ground, but also from the seismometers left on the Moon by the Apollo missions. These instruments record between 75 and 150 impacts per year from objects ranging from $100 \mathrm{~g}$ to $1000 \mathrm{~kg}$. Artificial impacts observable from the Earth are of major interest, since in these cases one knows in advance the nature of the impacting body. The most recent was the SMART-1 aircraft (Foing 2006), which crashed near the terminator area of the Moon on 2006 September 3 , and was observed with the WIRCam wide-field infrared camera

\footnotetext{
${ }^{1}$ See http://www.nasa.gov/centers/marshall/news/lunar/.
} 
from the $3.6 \mathrm{~m}$ Canada-France-Hawaii Telescope (CFHT) in Hawaii. ${ }^{2}$ For both natural and artificial collisions, the contrast with a dark background, for which the brightness has been precisely calibrated, will pave the way for further studies.

Continuous photometric observations of the earthshine reflected from the Moon have been carried out from Big Bear Solar Observatory (BBSO), in Southern California, since 1998. The primary goal has been to measure anomalies in the Earth's albedo, which requires high-precision instrumentation, a demanding nearnightly observing program, and meticulous data analysis. Here we use this data set to measure the surface brightness of the dark portion of the face of the Moon as seen from the Earth. This can be used to calibrate the visual magnitude of a variety of lunar impacts occurring sporadically, or during the course of meteor showers.

In the subsequent sections, we first explain the technique used to acquire the lunar data reported here. These data are usually taken to measure the Earth's albedo and to determine its anomalies. But besides this primary goal, the data can be used to calibrate the brightness of the lunar surface when illuminated by the terrestrial glow for various, evolving lunar phase angles. An estimate of the theoretical surface brightness is calculated in $\S 3$. Annual, seasonal, and daily deviations are explained in $\S 4$. The results for the calibration of brightness of the dark lunar surface by means of the data obtained during the lunar eclipse on 1993 November 29 are presented in $\S 5$. The results are reviewed in $\S 6$.

\section{METHODOLOGY: MEASURING THE BRIGHTNESS OF THE EARTHSHINE}

In previous publications, we have reported that we are able to detect variations in the global apparent albedo of the Earth of the order of $0.1 \%$ (Goode et al. 2001; Pallé et al. 2003, 2004). Our observing technique consists of taking images of the Moon with a $15 \mathrm{~cm}$ refractor, alternatively with and without an occulting filter to block the moonshine (sunlight reflected from the sunlit Moon). We take data through the night, as long as the Moon is up and the moonshine is small enough to allow a reliable background correction (this is for lunar phase angles [Sun-Moon-Earth] between $\pm 155^{\circ}$ and $\pm 40^{\circ}$ ). The local atmosphere is corrected by fitting the data to Beer's law and extrapolating the intensity to zero air mass.

In the reduction, we integrate the light from a pair of fiducial patches: one from the bright portion and one from the dark portion of the lunar disk. In our study, 10 physically fixed fiducial patches have been used, five near Grimaldi and five near Crisium. Their selenographic coordinates are as follows: $(-11.25,-71.50)$, $(-17.50,-70.00),(-5.00,-76.00),(0.00,-75.00)$, and (7.50, $-76.50)$ in the former case, and $(28.50,72.50),(12.50,75.00)$, $(0.00,77.00),(-7.50,75.00)$, and $(-13.00,75.00)$ in the latter case. The relative lunar reflectivity between each pair of patches has been measured by observing lunar eclipses and can be found in Table 4 of Qiu et al. (2003).

Here we use this data set to determine how the surface brightness of the earthshine varies with the Sun-Earth-Moon geometry and with the Earth's weather conditions. We have performed the usual steps in data reduction for our photometric images: dark current, flat-field, and bias corrections, and we have extrapolated the intensity values to zero air mass and carefully subtracted the background scattered light caused by the moonshine. The detailed steps of the data reduction were given in Qiu et al. (2003). Several corrections, such as the evolution of the lunar phase func-

\footnotetext{
${ }^{2}$ See http://www.cfht.hawaii.edu/News/Smart1/.
}

tion, lunar libration, and the opposition effect, are essential in determining the Earth's reflectivity. For the purpose of this paper, however, we can and have omitted these corrections.

The brightness of one fiducial region, $B_{\mathrm{ES}}$, can be calculated as follows,

$$
B_{\mathrm{ES}}=B_{C}+2.5 \log \left[\left(I_{C} / I_{\mathrm{ES}}\right)\left(a_{\mathrm{ES}} / a_{C}\right)\right],
$$

where $B_{C}$ is the surface brightness for the entire lunar crescent, $I_{C}$ is its total intensity, $I_{\mathrm{ES}}$ is the intensity for a given fiducial patch of the dark portion region, and $a_{\mathrm{ES}}$ and $a_{C}$ are the patch and the crescent areas, respectively. The $B_{C}$ value at zero air mass was determined from the Jet Propulsion Laboratory's HORIZONS ephemeredes generator. $^{3}$

The spectral coverage of our data is limited below $400 \mathrm{~nm}$ due to the quantum efficiency of the CCD camera used in our observations, and by two near-IR filters that cut all light above $700 \mathrm{~nm}$. The CCD, a back-illuminated Apogee 7, has a relatively flat transmission between 400 and $700 \mathrm{~nm}$. Our observations were not carried out with a Johnson's $V$ standard filter, which would transmit only $35.5 \%$ of the current flux; however, since we used relative measurements we have omitted any spectral correction. The wavelength-dependence of the terrestrial spectral albedo, basically the earthshine-to-moonshine ratio, has been previously studied for one night, 2003 November 19 (Montañés-Rodríguez et al. 2005).

\section{THEORETICAL ESTIMATES}

The brightness of the Moon's crescent can be easily determined, since it only depends on the geometry of the Sun-Earth-Moon system. But the brightness of the dark portion of the Moon depends, beyond changes associated with the Sun-Earth-Moon geometry, on the Earth's weather, which is unpredictable, and on the Earth's geography. A rough estimate of its brightness can be calculated as follows,

$$
B_{\mathrm{ES}}=B_{\mathrm{BS}} 2.5 \log \left(F_{T} / F_{S}\right) .
$$

Here $F_{T}$ and $F_{S}$ are the terrestrial and solar fluxes reflected from the Moon. To simplify our calculations, at a lunar phase angle of $90^{\circ}$ we find that

$$
B_{\mathrm{ES}}=5.25-2.5 \log \left[A\left(R_{\mathrm{E}} / d_{\mathrm{EM}}\right)^{2}\right] \text {, }
$$

where $A, R_{\mathrm{E}}$, and $d_{\mathrm{EM}}$ are the terrestrial albedo, the Earth's radius, and the Earth-Moon distance, respectively. For a terrestrial albedo of 0.3 and a mean Earth-Moon distance equal to 60 times the terrestrial radius,

$$
B_{\mathrm{ES}}=15.44 m_{V} \operatorname{arcsec}^{-2} .
$$

Variations in the Earth-Moon distance and in the Earth's albedo are of the order of $10 \%$. They introduce an uncertainty of $2.8 \%$ and $1.4 \%$, respectively, in the observed brightness. The major contribution to changes in the earthshine comes from the Earth's phase function. Paradoxically, the maximum brightness occurs during a total eclipse of the Sun. This value has been measured before; for instance, during the total eclipse of 1968 September 22 (Koutchmy \& Koutchmy 1974) it was measured in order to study the coronal aureola phenomenon, and it was equal to about $+13 m_{V} \operatorname{arcsec}^{-2}$

The minimum brightness of the dark side of the Moon occurs at lunar phase zero, during a total lunar eclipse, when only the

\footnotetext{
${ }^{3}$ See http://ssd.jpl.nasa.gov/horizons.cgi.
} 

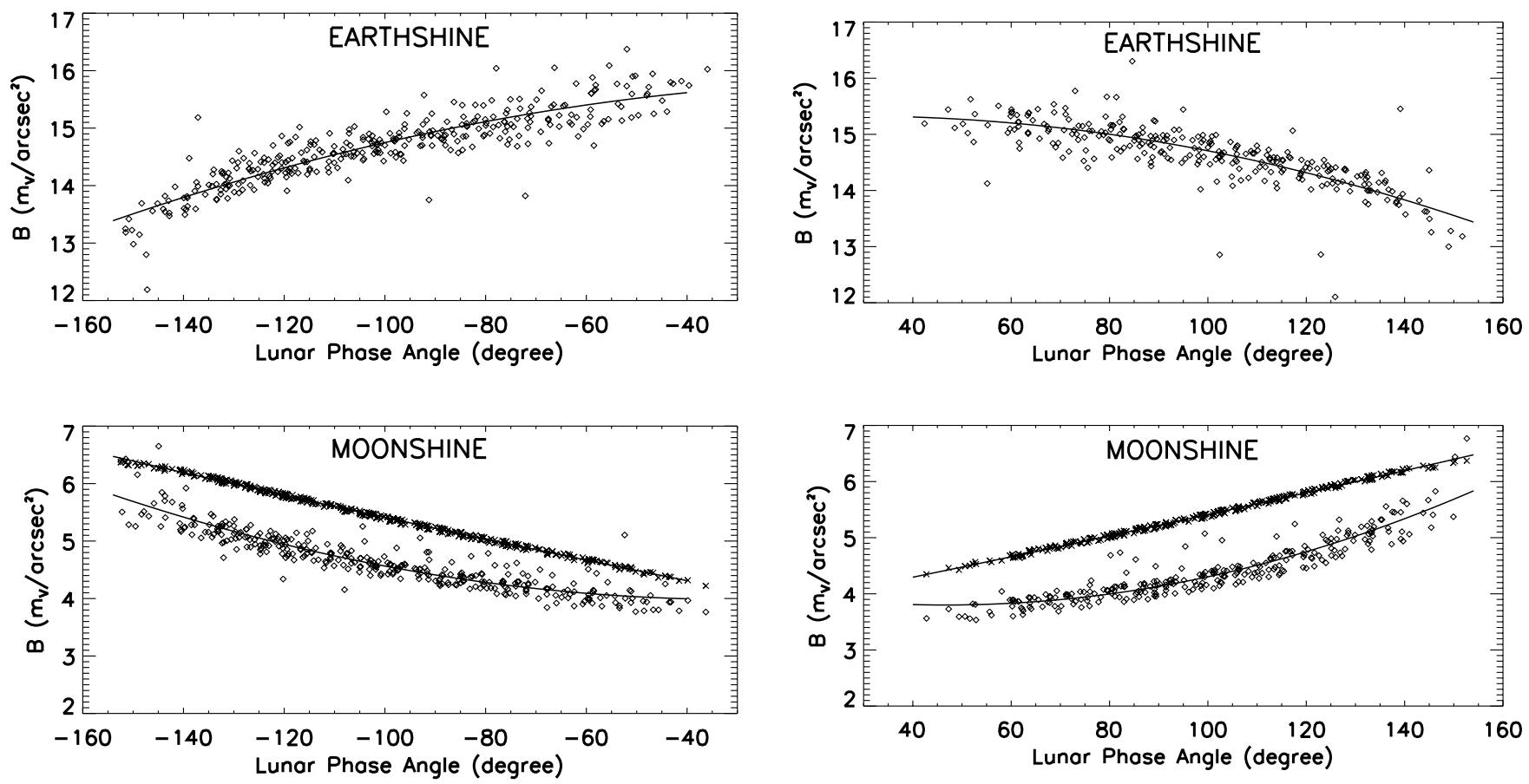

FIG. 1.-Mean daily value for the earthshine surface brightness at zero air mass (top) and for the moonshine and entire crescent (bottom), for negative (waxing Moon, left ) and positive (waning Moon, right) lunar phase angles. Data taken between 1998 and 2005 are shown and used in the fits. In the bottom panels, note the excellent fit for the crescent data and the major dispersion in the fit for the brightness measured from the fiducial patches (diamonds). This difference in brightness, of up to 1 mag, is because our selected patches are situated in the lunar highlands with a relatively higher reflectivity than the mean lunar reflectivity. The same difference is to be expected between the fiducial earthshine patches and the mean value for the entire dark region (not shown).

solar flux refracted around the Earth by the terrestrial atmosphere reaches the Moon.

\section{RESULTS FROM OBSERVATIONS}

We have determined the brightness of our 10 selected spots, five on the earthshine portion and five on the moonshine portion of the Moon, for our entire data set, from 1998 until 2005, covering all possible lunar phases. After averaging the brightness value for the five spots in the earthshine and in the moonshine, we have determined the best polynomial fits for positive and for negative lunar phases (Fig. 1).

All data taken between 1998 and 2005 have been considered, although about 20 nights with a deviation bigger than $3 \sigma$ were discarded from the earthshine fit, and four nights with a deviation bigger than $1 \sigma$ were removed from the bright-side fit. The discarded nights were not considered in any other fit thereafter.

Our results reveal that the surface brightness found from this fit is consistent with the predictions given in $\S 3$, although our observations do not cover all possible lunar phase angles. Phase angles larger than $155^{\circ}$ are not observed because the Moon is not visible long enough to allow a Beer's law fit, which is required to carry out a proper air-mass correction. Phase angles smaller than $40^{\circ}$ are not observable because the background scattered light is too strong to allow a reliable sky subtraction.

Figure 1 shows that the annual fit for the surface brightness varies with the lunar phase between 13.5 and $15.5 m_{V} \operatorname{arcsec}^{-2}$; this result is for the average of the five fiducial patches. For the moonshine region, we find that our spots are almost $1 \mathrm{mag}$ brighter than the entire crescent (crosses). Not surprisingly, the earthshine data have much more dispersion than the moonshine data. This dispersion is mainly caused by the lower signal-tonoise ratio of the earthshine data, but the real day-to-day changes in the Earth's albedo, driven mainly by the total cloud amount and the cloud properties on the sunlit Earth, also contribute.

\subsection{Annual and Seasonal Changes}

In order to distinguish among different possible physical causes for the changes in our brightness data, we have calculated three different fits to our data set. A daily mean value of the data was calculated for each night. Then, the data from the earthshine, the bright side, and the entire crescent were independently fit to a thirdorder polynomial (Fig. 1). A dispersion of up to $2 \mathrm{mag} \operatorname{arcsec}^{-2}$ is apparent in the earthshine fit, whereas a dispersion of less than $1 \mathrm{mag} \operatorname{arcsec}^{-2}$ is found from the bright-side patches. This is partially due to the considerably higher signal-to-noise ratio achieved in the observations of the bright-side patches compared to the dark-side ones (typically of about 1000 and 50, respectively). The data for the year 1999 were selected and grouped by seasons (Fig. 2). In each case they were fit to a third-order polynomial.

\subsection{Daily Changes}

The apparent terrestrial albedo, $A^{*}$, or the terrestrial albedo for a given angle of incidence, is a function of the $I_{\mathrm{ES}} / I_{\mathrm{BS}}$ ratio multiplied by several corrective functions (Qiu et al. 2003), where $I_{\mathrm{ES}}$ and $I_{\mathrm{BS}}$ are the intensities for a pair of lunar patches, one on the dark-side regions and another on the bright-side regions, respectively.

We can express $A^{*}$ in magnitude, $\left[A^{*}\right]$, as follows,

$$
\left[A^{*}\right]=-2.5 \log A^{*} \text {. }
$$

Changes in $\left[A^{*}\right]$ are due to the evolution of the cloud-geography pattern, over the sunlit Earth, during the observations.

Figure 3 shows a typical change in these patterns (top) and the measured albedo for the same nights (bottom). A significant decrease or increase in the detected albedo is observed when large free-of-cloud ocean areas (with very low albedo) or large optically 

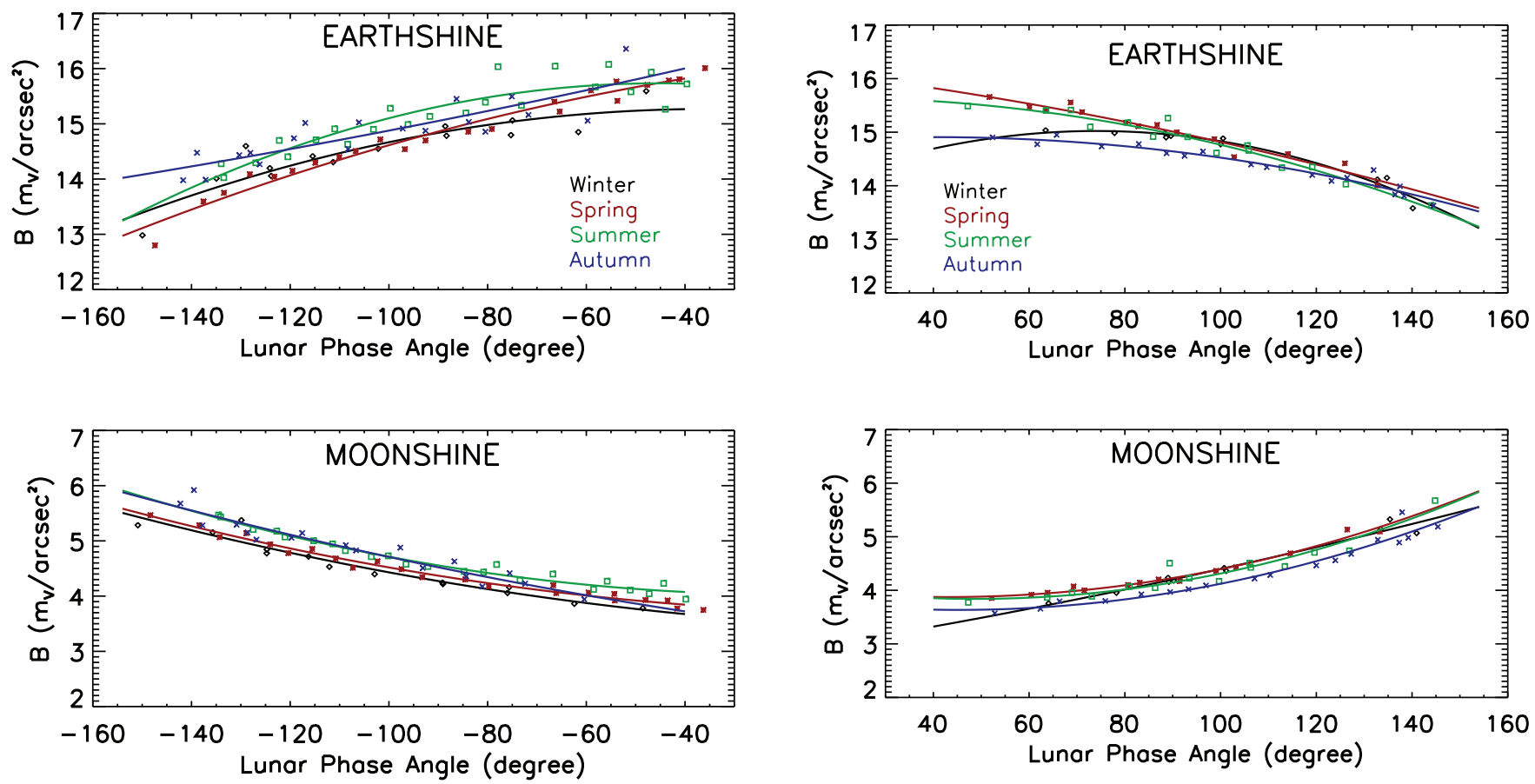

Fig. 2.- Same as Fig. 1, but here the data have been grouped by seasons. Different seasons are shown in different colors, and each season has been fit independently.

thick cloudy areas (with high albedo) appear, respectively, in the earthshine-contributing area of Earth.

\section{OBSERVED SURFACE BRIGHTNESS OF THE LUNAR GEOGRAPHY}

We have observed several lunar eclipses since the Earthshine Project started; the first was observed in 1993 November 23, using the $\mathrm{H} \alpha$ telescope in BBSO. This eclipse was observed over
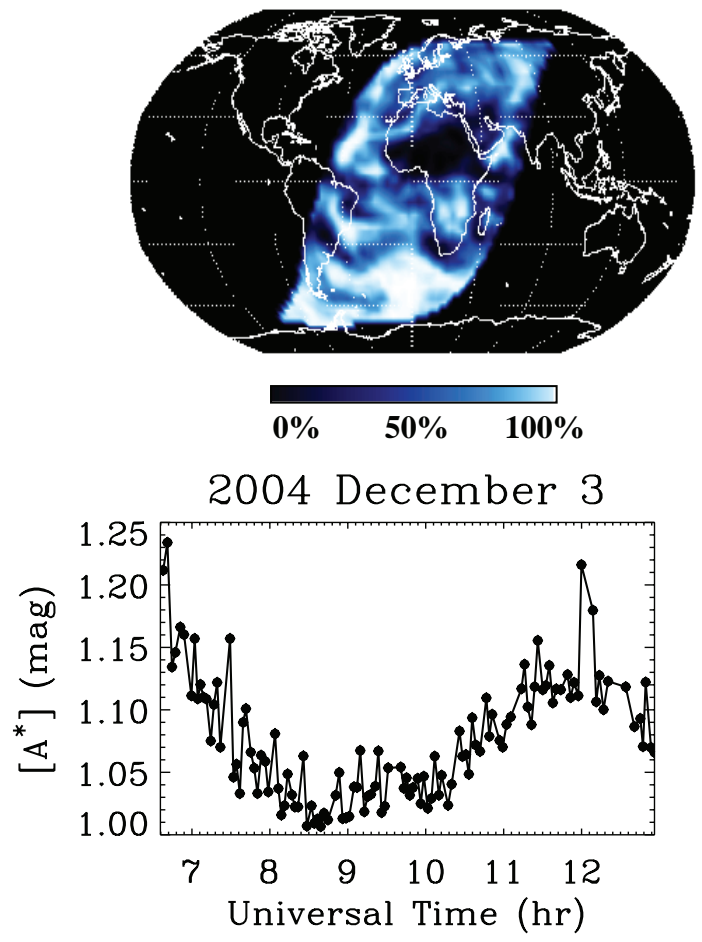

$11 \mathrm{hr}$, taking a cluster of several images every 30 minutes. Thus, a considerable number of photometric images, before and after the penumbra, are available (Montañés-Rodríguez et al. 2005). We have used all these images to interpolate an image at zero lunar phase angle, in order to obtain a flat, illuminated lunar map. Since the Moon's orbit has an axial tilt of $6.7^{\circ}$ with respect to the Earth's orbit plane, the illumination at phase angle zero is usually not perfectly flat for any eclipse. Thus, an additional subtraction
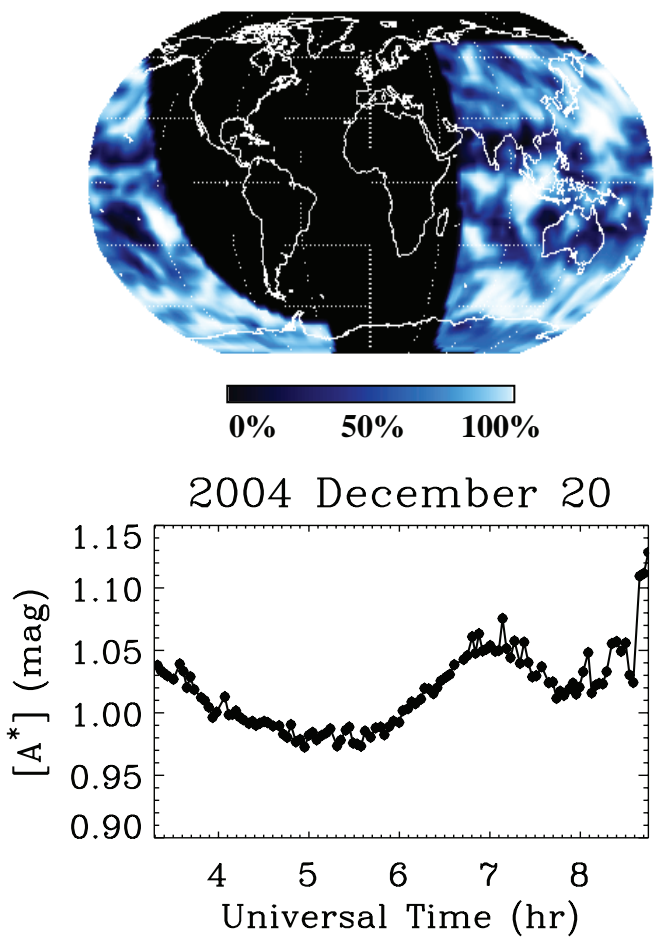

FIG. 3.-Top: Earthshine-contributing areas of the Earth for two nights, 2004 December 3 (left), with phase angle $-71^{\circ}$, and 2004 December 20 (right), with phase angle $+60^{\circ}$. Bottom: Apparent terrestrial albedo for these two nights. 

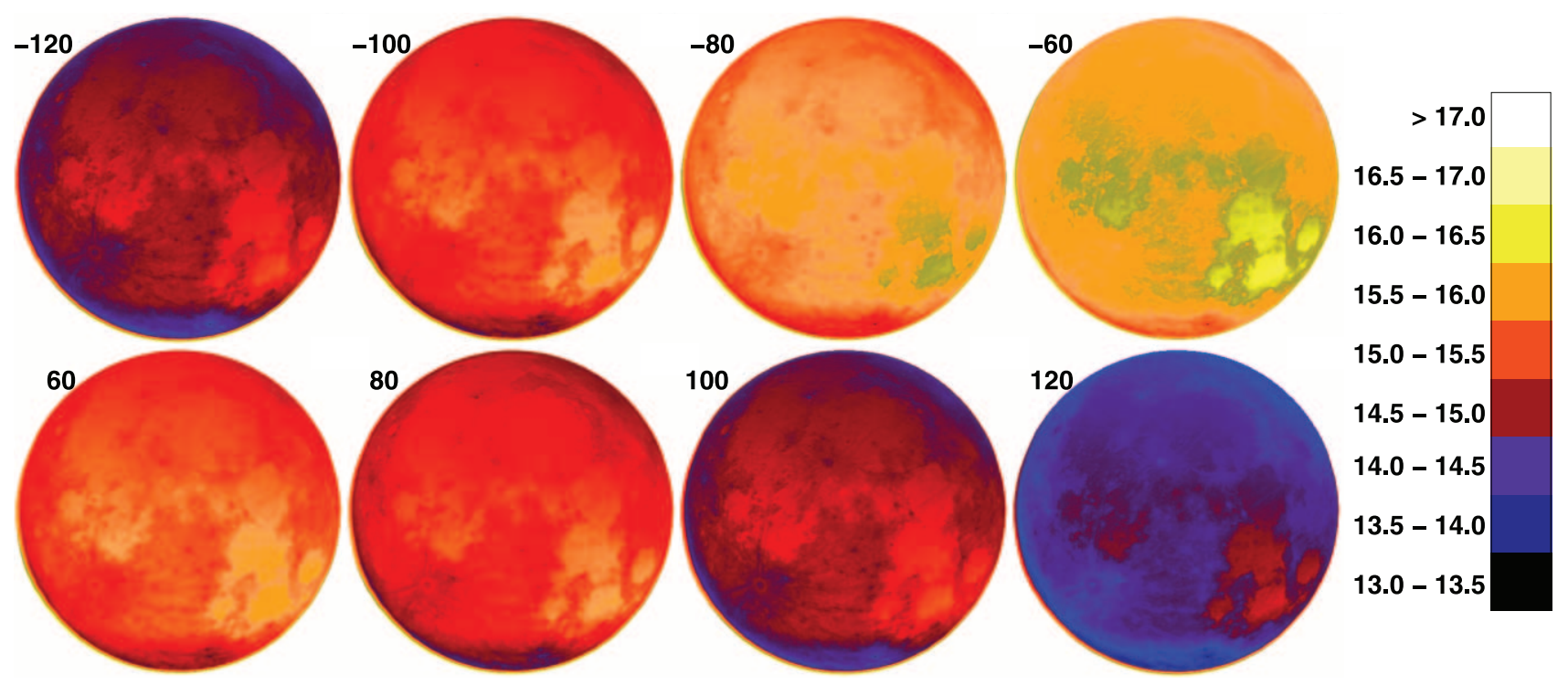

FIG. 4.- Lunar map calibrated in magnitude for different lunar phase angles. A similar map can be produced for each night of observations.

of the remaining illumination was made. The result of the interpolation and subtraction of this background is what we hereafter call an image at phase angle zero, $I_{0}$.

The value in magnitude of $I_{0}$ is useful for carrying out the relative calibration of lunar events. Events may, of course, take place at any lunar phase, not just at $0^{\circ}$. We used the fiducial patches previously described to interpolate the magnitude of $I_{0}$ at different lunar phase angles for the entire lunar map.

This calibration can be done more accurately for the day of a particular lunar impact event. However, and just to give an estimate of our precision, we show in Figure 4 the result for eight different lunar phase angles, determined from the annual fit to our five fiducial patches. We can achieve a better precision if we use the measured intensities for the fiducial patches instead of the annual fit. In the annual fit, in fact, the daily variations due to weather are neglected.

\section{CONCLUSIONS}

For this work, we have used our continuous nightly observations of the Moon, taken by alternatively measuring some wellcalibrated regions in the bright and dark lunar sides, to calculate the brightness of the lunar dark side. An approximation to the magnitude of the earthshine can be roughly estimated by calculating the incident flux in the same way as the brightness of the bright crescent is determined by taking into account the changes in the Sun-Earth-Moon system geometry. In fact, we used the theoretical brightness of the illuminated crescent to calibrate our observations of the dark side into $m_{V} \operatorname{arcsec}^{-2}$. However, the brightness of the dark side of the Moon has an unpredictable component that depends on the weather pattern over the sunlit Earth during the observations. Here we estimate the magnitude of this component to be of the order of $0.25 \mathrm{mag}$.

This accurate calibration of the earthshine's surface brightness had already allowed us to determine the brightness of several sporadic impacts between 7 and 8.3 visual magnitude (Ortiz et al. 2006). We also show here that if we consider this effect when we calibrate the lunar impacts, we can improve the calibration of $\sim 0.25 m_{V}$ arcsec $^{-2}$, provided the brightness of the earthshine is measured precisely, and its glow is subtracted from the brightness of any impact event.

The BBSO Earthshine Project is developing a robotic network equipped with $\mathrm{f} / 25$ refractor telescopes and Roper/Photometrics CCDs (Pallé et al. 2005). Although the short read-out time and pixel size of these CCDs ( $1 \mathrm{~ms}$ and $5^{\prime \prime}$, respectively) would allow the detection of lunar flashes, the robots were developed to cover the entire Moon and are not fast enough to permit the requisite resolution to detect a small lunar impact. However, the earthshine network will carry out continuous observations of the dark side of the Moon, from different locations on Earth, which can be used to perform a comparative calibration of nearly simultaneous lunar flashes. We intent to make these data available for that purpose.

This research was supported by a grant from NASA (NNG 04GN09G).
Bellot Rubio, L. R., Ortiz, J. L., \& Sada, P. V. 2000a, ApJ, 542, L65

Foing, B. H., et al. 2006, Adv. Space Res., 37, 6

Goode, P. R., Qiu, J., Yurchyshyn, V., Hickey, J., Chu, M.-C., Kolbe, E., Brown, C. T., \& Koonin, S. E. 2001, Geophys. Res. Lett., 28, 1671 Koutchmy, O., \& Koutchmy, S. 1974, A\&AS, 13, 295

Montañés-Rodríguez, P., Pallé, E., Goode, P. R., Hickey, J., \& Koonin, S. E. 2005, ApJ, 629, 1175

Ortiz, J. L., Quesada, J. A., Aceituno, J., Aceituno, F. J., \& Bellot Rubio, L. R. 2002, ApJ, 576, 567

\section{REFERENCES}

Ortiz, J. L., et al. 2006, Icarus, 184, 319

Pallé, E., Goode, P. R., Montañés-Rodríguez, P., \& Koonin, S. E. 2004, Science, 304, 1299

2005, Geophys. Res. Lett., 32, 11803, DOI: 10.1029/2005GL022575 Pallé, E., et al. 2003, J. Geophys. Res., 108, 4710, DOI: 10.1029/2003JD003611 Qiu, J., et al. 2003, J. Geophys. Res., 108, 4709, DOI: 10.1029/2003JD003610 Zou, Y. L., Liu, J. Z., Liu, J. J., \& Xu, T. 2004, Chinese J. Astron. Astrophys., 4, 97 